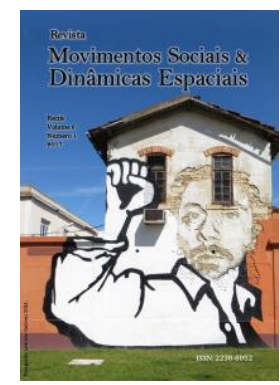

Revista

Movimentos Sociais \& Dinâmicas Espaciais

ISSN: 2238-8052

http://www.revista.ufpe.br/revistamseu

Artigo recebido em 13/05/2017 e aceito em 25/06/2017.

\title{
LA NOTION DE JUSTICE SPATIALE - UNE APPROCHE INSPIREE PAR LA PHILOSOPHIE DE JOHN RAWLS
}

A NOÇÃO DE JUSTIÇA ESPACIAL UMA ABORDAGEM INSPIRADA NA FILOSOFIA DE JOHN RAWLS

Bernard BRET 1

\begin{abstract}
RÉSUMÉ
Bien que sa procédure ait un fondement abstrait, mais parce qu'elle est rationnelle, la Théorie de la Justice du philosophe John Rawls énonce des principes précieux pour une approche géoéthique des territoires : le maximin qui permet de qualifier les inégalités territoriales au regard de la justice, l'égalité des personnes qui permet d'apprécier le territoire politique au regard de la démocratie, le principe de réparation qui doit inspirer l'aménagement du territoire en vue d'y promouvoir la justice sociale.
\end{abstract}

Mots-clés: géoéthique, justice spatiale, maximin, modèle centre-périphérie, Rawls.

\section{RESUMO}

Embora seu procedimento possua um fundamento abstrato, mas que se trata de um procedimento racional, a Teoria da Justiça do filósofo Jonh Rawls enuncia princípios preciosos para uma abordagem geoética dos territórios: o maximin que permite qualificar as desigualdades territoriais com relação à justiça, a igualdade das pessoas que permite apreciar o território político com relação à democracia, o princípio da « reparação » que deve inspirar o ordenamento territorial visando à concretização da justiça social no território.

Palavras-chave: geoética, justiça espacial, maximin, modelo centro-periferia, Rawls.

1 Professeur émérite, Université Jean Moulin, Lyon UMR EVS 5600 du CNRS. Mail: bernard.bret12@wanadoo.fr. 


\section{INTRODUCTION}

Le terme de justice spatiale s'est imposé depuis quelques années. Le fait traduit chez les géographes la conscience que leur activité de recherche comporte des implications d'ordre éthique, dans les thèmes qu'ils traitent, les méthodes qu'ils suivent et, éventuellement, les choix d'aménagement qu'ils inspirent. Est-ce à dire que la notion soit bien identifiée par tous et repose sur un ancrage théorique solide? Peut-être pas, et certainement pas toujours. Le présent article vise donc à clarifier cette question.

Une précision s'impose d'emblée: la justice spatiale n'est pas une justice entre les lieux, ce qui n'aurait à proprement parler pas grand sens, mais elle est la composante spatiale de la justice sociale. La perspective dans laquelle se situe la géographie est en effet le socio-spatial, à partir du constat que le social agit sur le spatial et que le spatial réagit sur le social. Ainsi, exemple bien connu parmi mille autres possibles, l'inégalité des revenus provoque la ségrégation sociale dans l'espace urbain, et en retour, par un effet de lieu, vivre dans un quartier défavorisé stigmatise les résidents et les met en état d'infériorité par rapport aux autres citadins. Une approche géoéthique des territoires apparaît dès lors utile. Elle voit le territoire pour ce qu'il est, un produit social, et s'autorise à qualifier son organisation au regard de valeurs éthiques.

Néanmoins, se pose immédiatement le problème de la légitimité d'une démarche associant une prétention scientifique avec des appréciations normatives de nature éthique. On pourrait lui objecter la nécessaire neutralité axiologique du discours scientifique. Le problème est ancien. Il y a déjà plus d'un siècle, le sociologue allemand Max Weber [ ] distinguait deux registres qui ne devaient pas se mélanger, d'une part le registre du savant dont la tâche est de comprendre le monde, y compris dans les sciences sociales, et d'autre part le registre du politique qui est celui de l'action dans le monde. Que le responsable politique agisse sur le réel en fonction de certaines valeurs, soit. Mais, en revanche, que le savant introduise ces valeurs dans ses analyses pose problème car il risque d'adopter un positionnement partisan. On comprend la difficulté, mais on s'interroge aussi sur la possibilité de rester neutre devant les situations qui choquent l'intuition de justice présente en chacun de nous. Rester neutre dans l'analyse des inégalités extrêmes, d'un côté l'opulence et de l'autre la misère, d'un côté de luxueuses villas et de l'autre des favelas, d'un côté de grands propriétaires fonciers et de l'autre des paysans sans terre, n'est-ce pas se montrer insensible à l'injustice du monde? Ne pas la dénoncer, n'est-ce pas se rendre complice de la domination que subissent les plus pauvres?

Comment sortir de cette double impasse? Décrire les faits sans rien en dire de plus et paraître cautionner des injustices que nous réprouvons? Dénoncer ce qui nous apparaît comme 
choquant, au risque alors d'abandonner son statut de chercheur et suivre une intuition de justice certainement respectable mais dépourvue de crédibilité scientifique?

\section{LA THÉORIE DE LA JUSTICE DE JOHN RAWLS}

Une solution à ce dilemme est ici proposée, consistant à nourrir l'analyse des territoires d'une conception de la justice explicitement revendiquée. Mieux vaut, en effet, afficher l'idée que l'on se fait de la justice et penser en fonction de ce que cette idée autorise à dire. Dans ces conditions, et si l'ancrage théorique est fondé en raison, la démarche n'appelle pas le soupçon de taire des préalables susceptibles d'invalider le raisonnement, ni le soupçon de reposer sur une intuition éventuellement fragile et qui ne ferait pas nécessairement consensus.

La thèse ici défendue est que la Théorie de la Justice du philosophe John Rawls [1971, 1987] offre un cadre efficace pour une géoéthique. Elle n'est pas la seule, bien entendu, qui apporte réponse aux interrogations du monde, mais il vient d'être dit qu'il faut afficher sa référence théorique: le présent article se réclame explicitement de la théorie rawlsienne. Que John Rawls ne parle jamais d'espace n'est pas une difficulté car la robustesse d'une théorie philosophique se vérifie précisément à sa capacité à nourrir tous les champs de la pensée. Par ailleurs, si, pour être recevable dans une démarche à prétention scientifique, l'idée de justice doit elle-même être validée par un fondement rationnel, tel est bien le cas chez John Rawls dont la théorie repose sur la raison et elle seule.

John Rawls s'inscrit dans la tradition des philosophies du contrat dont la figure emblématique est Jean-Jacques Rousseau. Par contrat, il faut entendre contrat social, c'est-à-dire contrat entre les hommes sur les règles du vivre ensemble. Un tel contrat, fût-il implicite, est indispensable, puisque les hommes sont par définition des partenaires sociaux (aucun d'eux ne peut vivre seul et chacun a besoin des autres) en concurrence les uns avec les autres. Des règles acceptées par tous doivent en conséquence fixer les droits et les devoirs de chacun avec l'objectif que fonctionne l'interaction sociale. Comment fixer les termes du contrat social? Sans exposer en détail la théorie rawlsienne - ce serait impossible en quelques pages et cela nous écarterait de notre but - il vaut la peine de dire qu'elle prétend dire des principes ayant valeur universelle et que, pour ce faire, elle adopte une procédure d'énonciation purement abstraite. John Rawls constate que la diversité des hommes empêche d'imaginer une structure où tous les partenaires sociaux vivraient dans une stricte égalité sur tous les plans. On peut le regretter, mais un tel regret n'aboutit à rien d'autre qu'à la démission de la pensée et à la paralysie de l'action. Mieux vaut examiner comment répartir équitablement les coûts et les avantages de la coopération sociale. Pour ne pas être juge et partie dans cette affaire, il faut prendre une distance d'avec la 
position sociale que l'on occupe dans le monde. John Rawls appelle cette distance le voile d'ignorance, lequel n'a évidemment aucune réalité physique et désigne le positionnement méthodologique à adopter. Derrière le voile d'ignorance, je ne sais rien du monde réel si ce n'est qu'il y a des inégalités, mais en ignorant la place que j'y occuperais : je sais qu'il y a des riches et des pauvres, mais j'ignore si j'y serais riche ou pauvre, je sais qu'il y a des hommes et des femmes, mais je ne sais pas si le hasard me ferait naître fille ou garçon, je sais qu'il y a des handicapés et des personnes en bonne santé, mais je ne sais pas quel serait mon état de santé. Dans ces conditions où je ne sais rien du sort qui serait le mien tout en sachant que certains seront mieux lotis que d'autres, la réponse rationnelle consiste à dire qu'il faut donner le plus possible à ceux qui auront le moins. Il serait irrationnel de répondre autrement car il peut se faire que, dans le monde réel, je me trouve dans la catégorie la plus défavorisée. John Rawls aboutit donc au principe de justice qui veut donner le plus possible à ceux qui ont le moins, c'està-dire à maximiser la part de ceux qui ont le moins, c'est-à-dire encore à maximiser le minimum, principe résumé sous le terme de maximin.

S'il est apparu indispensable de dire quelques mots sur la façon dont John Rawls procède pour énoncer les principes de justice, c'est parce que sa méthode heurte de front la tradition empirique préférée souvent en géographie. Pour beaucoup de géographes, il faut partir des faits, du concret pour ensuite, dans un deuxième temps, monter en généralité de façon à produire une géographie dite précisément géographie générale qui met en évidence les régularités que présentent en commun les cas observés. Une telle géographie va donc du particulier au général. La démarche rawlsienne est radicalement opposée à cette façon de faire. Sa procédure part de l'abstrait pour aller au concret et énonce l'universel pour analyser le particulier. Elle ne doit pas être mal comprise: partir de l'abstrait ne signifie pas que l'on partirait de rien, mais que, en tant qu'être rationnel et derrière le voile d'ignorance, on fait appel à la raison et à elle seule pour dire ce qu'est le juste, et ensuite revenir dans le monde réel avec les principes qui permettront de le comprendre.

Le but ici poursuivi est de montrer que cette démarche rawlsienne constitue un cadre précieux pour l'analyse géographique parce qu'elle offre une grille de lecture pour interpréter les territoires et qualifier leur organisation au regard de principes éthiques.

\section{LE MAXIMIN ET L'ESPACE DU DÉVELOPPEMENT INÉGAL}

L'utilité du maximin en géographie tient en ce que ce principe permet de répondre à une question délicate touchant le développement. Il met en cohérence deux assertions qui paraissent exactes prises séparément et qui peuvent paraître contradictoires prises ensemble. La première 
dit que le développement est la croissance plus la justice. La seconde constate que le développement est nécessairement inégal. Non seulement le développement ne supprime pas toutes les inégalités, mais encore il en crée. De fait, on n'a jamais vu un développement se produire de façon uniforme et on a quelque difficulté à concevoir cette éventualité sur le plan théorique. Cela invite à considérer que la justice et l'égalité entretiennent des relations plus complexes qu'on ne l'imaginerait de prime abord Tout développement produit des inégalités entre les personnes, entre les branches d'activités et entre les lieux. Le modèle centre-périphérie rend compte de la transcription géographique de ce phénomène. Comme on le sait, la richesse se concentre en certains points parce que c'est là que se concentrent aussi les facteurs de production, les hommes, les capitaux, le pouvoir de décision économique et souvent politique, à quoi s'ajoutent en bien des cas la création intellectuelle et artistique. Par opposition, la périphérie a des activités économiques banales et offre des conditions de vie, en moyenne, inférieures à celles du centre. Il y a donc inégalité. Y a-t-il pour autant injustice? C'est là que la notion de maximim est précieuse. Si le centre prélève dans la périphérie tout ce dont il a besoin, les hommes, les capitaux, les ressources naturelles, et cela au point d'appauvrir la périphérie, il est clair que la configuration est injuste. En revanche, si le centre joue un rôle de pôle de croissance et entraîne la périphérie dans son développement, soit en distribuant la richesse produite en son sein, soit en diffusant dans la périphérie sa capacité à produire, la configuration n'est pas injuste. Elle n'est pas injuste dans le sens que les habitants de la périphérie bénéficient alors, en moyenne, de conditions de vie meilleures que s'il n'y avait pas le centre. Il faut donc bien distinguer le centre qui a un rôle de prédateur et le centre qui a un rôle de locomotive, pour reprendre l'expression souvent utilisée pour qualifier le rôle de São Paulo à l'égard du territoire brésilien. Dans un ouvrage pionnier, Alain Reynaud [1981] avait ainsi distingué d'une part la périphérie exploitée, devenant périphérie délaissée après que toutes ses richesses ont été pillées et qu'elle ne présente donc plus aucun intérêt pour le centre, et d'autre part la périphérie intégrée, c'est-à-dire la périphérie qui participe au dynamisme économique du centre et qui, sans avoir les pouvoirs de décision, reçoit des investissements et des emplois.

Ainsi en est-il du territoire brésilien. Il y a un centre économique indéniable, la ville de São Paulo, dont les fonctions financières et de commandement renforcent chaque jour son rôle de métropole et son statut de ville mondiale. Il y a une région périphérique, le Nordeste, longtemps exploitée puis délaissée, et maintenant en cours d'intégration depuis que l'Etat y favorise le déploiement des capitaux paulistes. Il y a aussi autour de la capitale économique une vaste région dépassant l'Etat de São Paulo et qui participe du dynamisme pauliste. Mais, à l'intérieur même du centre, résident des pauvres dont beaucoup sont venus de la périphérie et qui tirent de leur proximité géographique avec la richesse des retombées en termes d'emplois et de services publics. Changer l'échelle d'observation comme il vient d'être fait suggère alors 
qu'un centre peut avoir à la fois un rôle prédateur sur un vaste territoire, et un rôle d'entraînement sur un territoire plus restreint. En s'autorisant d'abord un pléonasme et ensuite un oxymore, on peut alors distinguer une périphérie externe et une périphérie interne. La première est exploitée alors que la seconde tire avantage de son insertion dans le centre. Ce sont là choses connues sur lesquelles on ne s'attardera pas, mais qu'il fallait rappeler pour la suite du raisonnement, non sans ajouter que des configurations comparables se trouvent ailleurs dans le monde. Le Brésil est une illustration d'un modèle général d'organisation de l'espace, mais illustration que le contraste entre la richesse et la pauvreté rend particulièrement démonstrative.

Le phénomène se vérifie à toutes les échelles géographiques, raison pour laquelle un modèle peut en rendre compte. On le montrera aux deux échelles extrêmes, celle de la planète et celle de l'espace privé. A l'échelle de la planète, la mondialisation a transformé la géographie des lieux de pouvoir et de production de richesse, sans invalider toutefois l'opposition Nord-Sud. Parce qu'ils offrent des conditions de vie supérieures en moyenne à ce que connaissent les pays du Sud, les territoires pauvres du Nord constituent une périphérie interne. Ceux des pays du Sud qui n'arrivent pas à décoller économiquement sont, eux, la périphérie externe, c'est-à-dire exploitée. Le transfert de richesse réalisé de la périphérie externe vers le centre (les prix du commerce international, les flux de main-d'œuvre) participe des retombées dont bénéficie la périphérie interne des pays du Nord: intrication étroite du fait social avec le fait spatial. A l'échelle de l'espace privé, la condition des employés de maison, les empregadas au Brésil, montre que la même logique se vérifie. L'empregada est évidemment dans une situation sociale d'infériorité et de dépendance par rapport à son employeur. Elle loge dans le quarto da empregada qui est une sorte de périphérie par rapport au reste de l'appartement ou de la maison où elle travaille. Néanmoins, sur le strict plan matériel, l'empregada est dans une situation meilleure que celui ou celle qui n'a pas trouvé d'emploi et qui habite un quartier inforrmel dépourvu des équipements de base. Les domestiques des riches constituent ainsi une sorte de périphérie interne dont le sort ingrat peut malgré tout être envié par ceux qui se trouvent dans la périphérie externe, de même que le salarié, fût-il mal payé, peut être envié par le chômeur.

Comment qualifier cela au regard de l'éthique? Il est clair qu'aucune de ces situations n'est juste car aucune n'est conforme au principe du maximin. Au demeurant, une configuration géographique ne pourrait être juste que si elle était produite par une structure sociale ellemême juste, ce qui n'existe nulle part. Du moins, peut-on considérer que les configurations géographiques sont plus ou moins justes, et pour en juger, il est nécessaire de déterminer ce qu'est le juste selon une procédure rationnelle et abstraite antérieure à l'observation des faits. 
La démarche pluriscalaire coutumière aux géographes examine alors si une plus grande justice obtenue en un lieu est ou n'est pas financée par l'aggravation de l'injustice à une autre échelle.

\section{LE PRINCIPE D'ÉGALITÉ : RÉFLEXION SUR LE TERRITOIRE POLITIQUE}

De ce qui précède et en matière de justice distributive, on retiendra que si les inégalités sont le plus souvent des injustices, elles ne le sont pas nécessairement et comme par définition. Mais, la justice ne se réduit pas à la justice distributive. Certes, il est fondamental que la répartition des richesses et des positions sociales soit le plus équitable possible. Mais John Rawls énonce un autre principe qu'il place même au-dessus du maximin: l'égalité de la valeur intrinsèque des personnes, c'est-à-dire de leur valeur en soi. Cela veut dire que tous les partenaires sociaux ont une même valeur d'existence, malgré l'inégalité de leurs revenus respectifs et de la place qu'ils occupent dans la société. De fait, derrière le voile d'ignorance, personne ne pourrait rationnellement accepter que certains soient l'objet de mépris ou privés de leur liberté du fait de leur sexe, de leur couleur de peau, de leur origine ou d'autres facteurs de cet ordre, car personne ne sait si, dans le monde réel, le hasard ne le placerait pas dans le groupe ainsi discriminé. La liberté et l'estime de soi figurent donc parmi les valeurs fondamentales du système rawlsien. Dans l'ordre politique, l'égalité de la valeur d'existence des personnes signifie que chacun est un citoyen, ce qui valide la démocratie comme seul système juste de gouvernement.

Ce point concerne-t-il la géographie? Oui, sans aucun doute! Il s'agit alors d'examiner le territoire politique pour voir s'il permet ou non le bon fonctionnement de la démocratie. Comme il l'a fait pour le maximin, John Rawls nous invite à concentrer notre attention sur les structures de base de la vie en société. Parce qu'il s'agit d'une théorie politique de la justice, l'important tient dans les institutions car, en amont des comportements individuels, ce sont elles qui permettent ou empêchent la justice entre les individus. La perspective géographique retiendra alors le maillage politico-administratif du territoire comme une institution et posera la question suivante: l'architecture institutionnelle des territoires respecte-t-elle ou bafoue-t-elle le principe "un homme, une voix" qui constitue la base du système démocratique. En effet, que vaut ce principe si, au terme des opérations électorales, tous les suffrages n'ont pas le même poids statistique pour l'attribution des sièges à pourvoir? On n'exposera pas ici les systèmes électoraux, ni on ne plaidera en faveur du système proportionnel ou du système majoritaire. Chacun de ces systèmes a ses avantages, le système proportionnel respecte mieux les choix des électeurs et le système majoritaire garantit mieux la stabilité des gouvernements, et ses inconvénients, la difficulté à trouver des majorités nettes dans le premier cas et la non- 
représentation des opinions très minoritaires dans le second. Limitant le propos à ce que la géographie peut dire du sujet, on signalera seulement comment le découpage des circonscriptions électorales peut déformer l'expression du vote et porter atteinte au principe de l'égalité citoyenne. Il s'agit de la taille des circonscriptions électorales et de leur forme. Si une circonscription compte beaucoup plus d'électeurs inscrits qu'une autre pour un même nombre de sièges à pourvoir (un seul siège par circonscription dans un système majoritaire), il est évident que le poids statistique de chaque voix y sera inférieur à ce qu'il est dans la circonscription voisine: celui qui vote dans une circonscription comptant 50.000 inscrits est en quelque sorte deux fois plus citoyen que celui qui vote dans une circonscription comptant 100.000 inscrits! Par le biais du scrutin à deux degrés où les électeurs de base désignent les grands électeurs qui, eux, émettent le vote final, cet effet déformant a joué à plein lors des élections présidentielles de 2016 aux Etats-Unis qui ont vu la victoire de Donald Trump alors qu'Hillary Clinton avait un avantage de presque trois millions de voix! Le problème existe en maints autres pays. Ni la France, ni le Brésil n'y échappent. Pour Jacques Lévy [1994], résumant de façon savoureuse ce genre de situation, cela consiste à faire voter des kilomètres carrés. A ce problème de taille s'ajoute le problème de la forme, bien connu des politologues sous le nom de gerrymandering et de l'opinion publique sous le nom plus trivial et plus évocateur de charcutage électoral: le découpage partisan, et donc parfois tortueux, des territoires électoraux conçus pour favoriser un camp.

Aller plus avant que ces notations rapides de géographie électorale dépasserait l'objectif visé. Il s'agissait simplement de montrer l'importance qu'a la morphométrie (la forme et la dimension) du territoire politique pour la démocratie entendue comme la figure citoyenne de la justice. Deux remarques encore, toutefois. La première pour dire que le maillage politicoadministratif est important aussi pour la justice distributive quand il s'agit de répartir les prélèvements fiscaux (une des coûts de la coopération sociale selon les termes de John Rawls) et la dépense publique (un des avantages de la coopération sociale). La seconde pour procéder à un changement de focale et constater qu'un problème comparable, non pas identique mais comparable, se pose à l'échelle du monde, chaque territoire étatique ayant sa propre législation fiscale et sociale. Le maillage politique de la planète produit l'injustice entre les populations selon le hasard de leur lieu de naissance, et cela d'autant plus que le maillage est fin. Le cas extrême est celui des micro-Etats paradis fiscaux (tous les micro-Etats ne sont pas des paradis fiscaux, mais beaucoup le sont) assurant à leurs citoyens un bien-être financé artificiellement par les prélèvements opérés aux dépens des autres pays, outre qu'ils bénéficient d'une reconnaissance juridique leur valant dans le système international un poids sans rapport avec l'effectif de leur population. 


\section{LE PRINCIPE DE RÉPARATION ET L'AMÉNAGEMENT DU TERRITOIRE}

$\mathrm{Si}$, derrière le voile d'ignorance, la théorie rawlsienne refuse de considérer par définition toute inégalité comme une injustice, ce n'est pas pour nier les injustices du monde réel, mais, bien au contraire, pour être capable de qualifier d'injustes les situations qui lui apparaissent telles. Qu'est-ce alors qu'une injustice, selon John Rawls? C'est une situation qui contrevient à un des deux principes de justice précédemment énoncés, ou aux deux à la fois: une inégalité qui contrevient au maximin, un non-respect de la valeur d'existence des personnes. Et alors, force est de le constater, l'injustice est partout dans le monde, sous une forme plus ou moins grave. Que faire dans ces conditions? La réponse rawlsienne est claire: réparer l'injustice. Comment procéder? En donnant plus à ceux qui ont moins, c'est-à-dire en pratiquant ce que les NordAméricains appellent affirmative action et les francophones la discrimination positive.

Là encore, notre réflexion se nourrit de la philosophie pour en voir les prolongements possibles dans la gestion de l'espace. Le principe de réparation prend ici la forme de l'aménagement du territoire entendu comme la mise en cohérence de l'organisation des lieux avec un projet de société. Donner plus à ceux qui ont moins pour viser le maximin, c'est localiser les infrastructures physiques et les services publics d'une façon telle que les usagers les moins bien desservis le soient le mieux possible. C'est aussi intervenir par des dispositions particulières en faveur des territoires jugés défavorisés, les mesures dérogatoires portant notamment sur les impôts et sur les aides à l'investissement et à l'emploi. Une fois le principe rappelé, il est inutile de détailler des dispositifs bien connus. C'est ce que l'Italie a fait en faveur du Sud avec sa Cassa per il Mezzogiorno, le Brésil en faveur du Nordeste avec la Sudene (Surintendência do Desenvolvimento do Nordeste) et la France avec la Datar (Délégation à l'Aménagement $d u$ Territoire et à l'Action Régionale) aujourd'hui englobée dans le CGET (Commissariat Général à l'Egalité des Territoires).

Pour résumer d'un mot le sens de toutes ces interventions, disons qu'elles sont des discriminations positives à base territoriale: délimitation d'un périmètre d'intervention et application à l'intérieur de la zone ainsi définie de conditions spécifiques dérogatoires du droit commun. C'est là que l'affaire se complique et ne va pas sans danger. L'objectif affiché étant de combattre des inégalités considérées comme injustes, le moyen utilisé est d'agir directement sur les lieux pour agir indirectement sur les hommes. Mais les territoires cibles ne sont pas habités par des personnes jouissant de conditions de vie comparables et ayant des intérêts sinon identiques, du moins convergents. Bien au contraire, si une région est pauvre en moyenne, c'est toujours que cette moyenne cache des inégalités fortes entre les habitants, le grand nombre de pauvres s'expliquant en partie par la domination qu'ils subissent de la part d'un petit nombre de 
riches.... Comment expliquer la misère des paysans sans terre et des minifondistes sans dire la concentration de la propriété entre les mains d'une oligarchie foncière? Ou le chômage de masse sans dire que les détenteurs de capitaux choisissent d'investir ailleurs? Ou l'habitat informel et les taudis sans mentionner la spéculation immobilière?

Dans un contexte de forte inégalité sociale, ce qui est en général le cas des régions pauvres, le risque existe que les groupes sociaux dominants détournent à leur profit les avantages particuliers concédés au territoire qu'ils habitent et qu'ils contrôlent. C'est ce qui s'est passé pendant longtemps dans le sertão semi-aride du Nordeste brésilien, et que les paysans victimes des accidents climatiques appelaient indústria da seca : l'Etat fédéral apporte de l'argent pour des travaux hydrauliques, les paysans sinistrés sont employés sur les frentes de trabalho, des travaux sont réalisés sur des terres non expropriées contrairement aux dispositions légales et finalement des réservoirs d'eau, les açudes, servent à l'abreuvement des bêtes des latifondistes et valorisent donc les grandes propriétés au lieu de servir à l'irrigation des champs des petits paysans. Ce scénario heureusement révolu aboutissait à ce qu'une politique affichant un but de justice territoriale consolidait en réalité l'injustice sociale. La politique d'industrialisation qui est venue ensuite n'appelle pas la même critique, mais les exemptions fiscales liées aux investissements dans le Nordeste ont servi les investisseurs paulistes au moins autant que les populations déshéritées de la région. Il est indéniable que cette politique a favorisé la croissance. Il est indéniable aussi qu'elle transformé le Nordeste, le faisant passer, pour reprendre la terminologie d'Alain Reynaud, d'un état de périphérie délaissée à celui de périphérie intégrée. Y a-t-il eu développement? Oui, dans une certaine mesure, si l'on en juge par les progrès des niveaux de vie, mais dans une dépendance forte à l'égard du pouvoir économique situé dans la métropole pauliste. Tout ici confirme que le développement est nécessairement inégal et laisse ouverte la question de l'équité du changement: le processus a-t-il amélioré autant que faire se peut le sort des plus pauvres de la région? Il est impossible de prétendre que l'on aurait atteint le maximin. On ne saurait nier pour autant que des progrès ont été réalisés. Est-ce juste ou injuste? Une telle question ne peut recevoir de réponse simpliste, car on est toujours dans le plus ou moins injuste et les arbitrages forcément réalisés dans les choix économiques et d'aménagement obligent à confronter les résultats atteints dans la région à ceux obtenus ailleurs et à d'autres échelles géographiques. Dire cela n'est pas se résigner à rester dans le flou, c'est tenir un discours qui colle à la complexité du réel.

Un point est sûr: il faut être très attentif aux conséquences sociales des politiques territoriales. Inversement, et parce que l'on est toujours dans le socio-spatial, il faut être également attentif aux conséquences territoriales des politiques sociales. Dans ses travaux récents sur la France, l'économiste Laurent Davezies [2008] démontre que les politiques sociales de redistribution y ont plus d'effets spatiaux que les politiques explicitement spatialisées ! Les 
transferts de revenus via les prestations familiales, les pensions de retraite, les allocations versées aux chômeurs et autres se traduisent aussi par des transferts spatiaux depuis les régions qui produisent plus qu'elles ne consomment vers des régions qui consomment plus qu'elles ne produisent. S'ajoute à cela un autre transfert spatial quand les consommateurs de passage dépensent dans les régions touristiques l'argent qu'ils ont gagné dans leur région de résidence. L'auteur parle d'économie résidentielle pour désigner ainsi des économies dont le moteur repose sur le pouvoir d'achat des habitants bénéficiaires des transferts de revenus et/ou sur les dépenses des touristes. Chiffres à l'appui, il montre qu'est totalement fausse la thèse selon laquelle, en France, la capitale exploiterait la province, idée souvent entendue depuis que JeanFrançois Gravier avait publié en 1947 un livre intitulé Paris et le désert français. En réalité, la région Ile-de-France produit plus de richesse qu'elle n'en consomme et redistribue le surplus à des régions moins dynamiques. C'est ainsi qu'une région comme le Limousin², dans le Massif central, a un faible PIB par habitant (elle produit peu) et un niveau très honorable pour les indicateurs de niveau de vie (elle consomme).

De même, il serait intéressant dans le cas brésilien de mesurer les conséquences locales et régionales de la Bolsa Familia, l'hypothèse étant que les territoires enregistrant les plus forts pourcentages de populations déshéritées ont vu leur économie stimulée par l'augmentation de la demande solvable. Distribuer du pouvoir d'achat aux plus pauvres, c'est relancer toute l'économie des territoires où habitent les populations bénéficiaires à travers les effets multiplicateurs des sommes introduites dans le circuit monétaire.

\section{CONCLUSION}

$\mathrm{Au}$ terme d'une réflexion à peine esquissée et qu'il faut évidemment poursuivre, trois idées simples mais fortes peuvent être avancées:

- Première idée, les sciences sociales sont légitimes quand elles incorporent la dimension éthique dans leurs travaux, à condition de dire explicitement leur référence théorique et de conduire leurs investigations en cohérence avec cette référence.

- Seconde idée, l'organisation du territoire est une entrée possible pour comprendre les faits sociaux, non pas la seule entrée, bien entendu, ni même sans doute la principale, mais une entrée possible à travers le socio-spatial. Tel est l'objet même de la géographie naguère qualifiée d'humaine, et aujourd'hui qualifiée de sociale, restant entendu qu'une géographie sociale au sens plein du mot est nécessairement aussi une géographie politique.

\footnotetext{
${ }^{2}$ Le Limousin, avec l'Aquitaine et le Poitou-Charentes, a été intégré à la région Nouvelle Aquitaine en 2016.
} 
- Troisième idée, enfin, par ses observations et ses diagnostics territoriaux, la géographie aide à repérer et à analyser les injustices, et en participant à l'aménagement du territoire, elle peut aider à produire le juste.

\section{BIBLIOGRAPHIE}

ANNALES DE GÉOGRAPHIE, Justice spatiale, $\mathrm{n}^{\circ}$ 665-666, janvier-avril 2009 (sous la direction de Philippe Gervais-Lambony).

BRET Bernard: Equité territoriale (définition), in Hypergéo, 2007.

BRET Bernard, GERVAIS-LAMBONY Philippe, HANCOCK Claire et LANDY Frédéric (dir): Justice et Injustices spatiales, Nanterre, Presses de Paris Ouest, 2010, 315 p.

BRET Bernard: Justice Spatiale, in: Géoconfluences, lettre d'information $\mathrm{n}^{\circ} 10$, Notions à la Une. septembre 2015.

BRET Bernard: Pour une géographie du juste, Lire les territoires à la lumière de la philosophie morale de John Rawls, Presses Universitaires de Paris Ouest, coll. Espace et Justice, 2015, $276 \mathrm{p}$.

DAVEZIES Laurent: La République et ses territoires. La circulation invisible des richesses, Paris, Le Seuil, "La République des Idées », 2008, 112 p.

DUFAUX Frédéric et Philifert Pascale (dir): Justice spatiale et politiques territoriales, Presses Universitaires de Paris Ouest, 2013, $324 \mathrm{p}$.

ESPACE GEOGRAPHIQUE (L'), Espace et justice sociale, $1978, \mathrm{n}^{\circ} 4$.

ESPACE POLITIQUE ( $\left.L^{\prime}\right)$, revue électronique (site: espacepolitique.revues.org)
FAINSTEIN Susan: The just city, Ithaca et Londres, Cornell University Press, 2010.

GUILLUY Christophe et NOYE Christophe, Atlas des nouvelles fractures sociales, Paris, Autrement, 2004, 63 p.

HARVEY David: Social Justice and the City, Baltimore, John Hopkins University Press, 1973, Londres, Arnold, 1973.

JUSTICE SPATIALE / SPATIAL JUSTICE, revue électronique bilingue (site: www.jssj.org)

LAURENT Eloi (dir): Vers l'égalité des territoires. Dynamiques, mesures, politiques, (Rapport au ministère de l'égalité des territoires et du logement), Paris, La Documentation française, 2013, $534 \mathrm{p}$.

LEFEBVRE Henri: Le droit à la ville, Paris, Anthropos, 1968, 165 p.

LEVY Jacques: L'espace légitime. Sur la dimension géographique de la fonction politique, Paris, FNSP, 1994, $442 \mathrm{p}$.

RAWLS John: Théorie de la Justice, Paris, Le Seuil, coll. La couleur des idées, 1987 (éd. originale 1971) $667 \mathrm{p}$.

REYNAUD Alain: Société, espace et justice, Paris, PUF, 1981, $263 \mathrm{p}$.

ZUCMAN Gabriel: La richesse cachée des nations, Enquête sur les paradis fiscaux, Paris, 2013, Le Seuil, " La République des Idées », $116 \mathrm{p}$. 